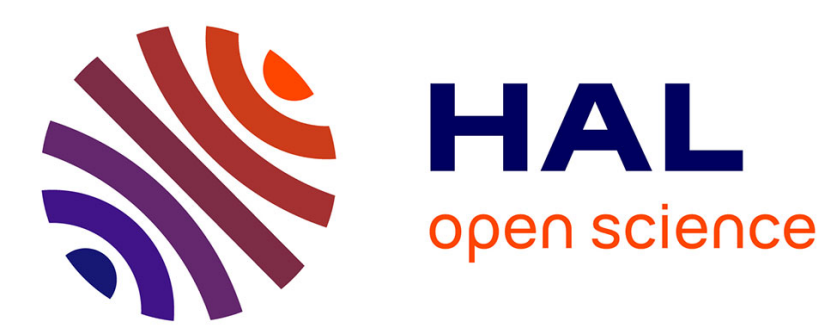

\title{
USE OF POLARIZATION ANALYSIS IN INELASTIC SCATTERING
}

\author{
F. Mezei
}

\section{To cite this version:}

F. Mezei. USE OF POLARIZATION ANALYSIS IN INELASTIC SCATTERING. Journal de Physique Colloques, 1982, 43 (C7), pp.C7-9-C7-22. 10.1051/jphyscol:1982702 . jpa-00222309

\section{HAL Id: jpa-00222309 https://hal.science/jpa-00222309}

Submitted on 1 Jan 1982

HAL is a multi-disciplinary open access archive for the deposit and dissemination of scientific research documents, whether they are published or not. The documents may come from teaching and research institutions in France or abroad, or from public or private research centers.
L'archive ouverte pluridisciplinaire HAL, est destinée au dépôt et à la diffusion de documents scientifiques de niveau recherche, publiés ou non, émanant des établissements d'enseignement et de recherche français ou étrangers, des laboratoires publics ou privés. 
JOURNAL DE PHYSIQUE

Colloque C7, supplément au $n^{\circ} 12$, Tome 43, décembre 1982

page $\mathrm{C} 7-9$

\section{USE OF POLARIZATION ANALYSIS IN INELASTIC SCATTERING}

\section{F. Mezei}

Institut Laue-Langevin, 156x, 38042 Grenoble Cedex, France

Central Research Institut for Physics, $P O B$ 49, 1525 Budopest, Hungary

Résumé - La polarisation neutronique peut être utilisée de deux manières dans la diffusion inélastique : d'une part pour l'identification sans ambiguîté des effets d'origine magnétique et, d'autre part, pour mesurer l'inélasticité elle même, indépendamment du caractère magnétique ou non-magnétique de la diffusion. Pour le premier type de ces applications, il $n^{\prime} y$ a que peu d'exemples, mais le nombre devrait augmenter rapidement du fait de l'intérêt croissant présenté par la diffusion diffuse. Par contre les techniques variées de modulation de polarisation, comme l'inversion rapide du spin et, en particulier, 1 'écho de spin neutronique, sont devenues des méthodes courantes dans le domaine de la diffusion inélastique. Le présent article couvre les principes de ces deux catégories d'utilisation des neutrons polarisés, ainsi qu'un certain nombre de résultats expérimentaux.

Abstract - Neutron polarization can be used in two ways in inelastic scattering : (a) for the identification of scattering effects of magnetic oxigin, and (b) as a tool to measure the inelasticity itself, independently of the magnetic or non-magnetic character of the scattering. There are only few examples for the first kind of applications, but this number is expected to increase rapidly with the growing interest in diffuse scattering. On the other hand, various polarization modulation techniques, such as spin-flip chopping and, in particular, neutron spin echo became by now rather routinely used methods in inelastic scattering. The present review covers the principles of both types of utilization of polarized neutrons, together with a number of experimental results.

1. Introduction - Before turning to the matter the title of this talk needs some explanation. Indeed, the expression "polarization analysis" is being used rather unprecisely and in more than one sense. Historically, the scattering of polarized neutrons on condensed matter was first considered as early as in 1937 by Halpern and Johnson, and in their amazing paper of 1939 they established the fundamental relation between the incoming and outgoing neutron polarization in scattering on. paramagnets [1] - at a time when it was only a speculation that the neutron might have a substantial magnetic moment. In this pioneering work the neutron spin polarization was considered as a vector, and its change in the scattering process was described as a vector-vector relation. The term "polarization analysis" (PA) should therefore mean the determination of a polarization vector, as it is done in experiments more frequently referred to as "vector polarization analysis" or "three dimensional polarization analysis". This latter type of work is most often done in beam transmission studies of magnetic domain structure and kinetics, and it has been pioneered by the Delft and Leningrad groups as early as 1969 [2,3]. Alperin has demonstrated in 1973 in an ad hoc fashion the feasibility of vector PA in crystallography [4], and a general experimental technique for doing this has been described by the author at the same time [5]. A further project on vector PA is in progress at the ILL [6]. Finally, the Halpern and Johnson equation is the basis of the application of neutron spin echo [7] to paramagnetic samples, which in fact provided the first full experimental test of this vector relation, as it will be shown in the present paper.

The most often used sense of the term "polarization analysis" is the one introduced in 1969 by the famous article of Moon, Riste and Koehler [8], in spite of these authors' warning of their improper use of these words : "(The title of the paper) 
is somewhat misleading, because we do not measure polarization at al1". In fact, the method proposed and tested in this work consists of the investigation of the single component of the scattered beam polarization which is parallel to the incoming beam polarization, which in turn is parallel to the magnetic field on the sample. This restriction of both the incoming and outgoing beam polarization to a scalar quantity, i.e. the component parallel to the field direction (which will be referred to in what follows as the $h$-component) is a vast simplification of the experimental procedure and still provides a very powerful tool for the identification of magnetic scattering effects.

Finally, if the scattering cross section depends on the h-component of the polarization one can either use a polarized incoming beam without analysing the polarization of the scattered beam or, alternatively, use an unpolarized beam and analyse the h-component of the scattered beam. These procedures are most often referred to as "scattering of polarized neutrons," rather than "polarization analysis".

In the present review I will consider all of these techniques in connection with inelastic scattering. In contrast to diffraction studies, in inelastic scattering the neutron polarization can be utilised not only for the unambiguous identification of the magnetic scattering effects but also as a powerful tool for measuring the scattering inelasticity itself. As a matter of fact, there are many more practical examples of this latter type of applications than of the first one. In many cases the investigated scattering is not at all of magnetic origin, in others the neutron polarization allows both the identification and the inelastic analysis of the magnetic effects.

In what follows we will first consider the relations describing the scattering of polarized neutrons in various, experimentally relevant situations, and review the experiments in which practical use was made of these relations. The second half of the paper is devoted to the description of the various inelastic scattering methods based on various schemes of polarization modulation and their applications. With the exception of neutron spin echo and its applications, which alone represent by now a bigger volume than all of the other topics together and will therefore be only very briefly invoked, the present review was aimed to be fairly complete.

\section{Scattering of polarized neutrons}

The most general case of scattering of polarized neutrons we are interested in both the scattering cross section and the polarization vector $\overrightarrow{\mathrm{p}}$. of the scattered beam for an incoming beam with an arbitrary polarization $\vec{P}$. Since Halpern and Johnson derived their classical equation [1]

$$
\vec{p}^{\prime}=-\vec{q}(\vec{q} \vec{p}) /\left|q^{2}\right|
$$

(where $\vec{q}$ is the neutron momentum transfer) for the scattering on ideal paramagnets, the theory has been gradually extended to samples of any complexity, in which the combination and interplay of magnetic, nuclear and nuclear spin scattering effects play an important role [9]. In the most general form the results become so complicated that only various simplified special cases are experimentally tractable. Much of the complexity comes from the interference between various types of contributions. In inelastic scattering these are generally small and do not provide too interesting information (in contrast to structural studies), except for the magnetovibrational scattering, i.e. the magnetic contribution to the phonon cross section. This scattering effect has exactly the same polarization dependence as the Bragg scattering, and it is discussed in Ralph Moon's paper in this volume. Here I will only consider purely magnetic scattering, but in practice, the caution has to be kept in mind that the magnetovibrational cross section is often not negligible.

The special cases which will be discussed below correspond to well defined experimental geometries and sample properties. I will use the code names "ferromagnetic" "paramagnetic" and "antiferromagnetic" situations, which evoke the fundamental features by the type of samples for which a given configuration and measuring procedure is the most typical. Nevertheless, as it will be shown below, with 
certain restrictions other kinds of samplescan also be investigated by each of these methods.

\section{A) Ferromagnetic situations}

Due to their high magnetization and domain structure, ferromagnetic samples tend to depolarize the impinging neutron beam unless they are magnetized to saturation [10]. Thus a strong magnetic field has to be applied to such samples, which makes any precessing component of the neutron beam polarization (i.e. those perpendicular to the field dixection) average very rapidly to zero. Consequently the polarization of the beam impinging on the sample, $\overrightarrow{\mathrm{P}}$ can only be parallel to the magnetic field $\vec{H}$, and only the $h$ component of the scattered beam polarization $\overrightarrow{\mathrm{p}} \mathrm{r}$ will be maintained and can be measured. Due to these restrictions we can describe the beam polarization by a single scalar quantity $\mathrm{P}$ :

$$
\mathrm{P}=\mathrm{p}_{\uparrow}-\mathrm{p}_{\downarrow} \quad\left(\mathrm{p}_{\uparrow}+\mathrm{p}_{\downarrow}=1\right)
$$

where $p_{\uparrow}\left(p_{\downarrow}\right)$ is the probability of occupation of the $\uparrow(\downarrow)$ state of the neutron magnetic moment with respect to the field direction taken as the z-axis. This is actually the configuration introduced by Moon, Riste and Koehler [8].

In this particular case, in view of eq.(2) the scattered beam polarization $\mathrm{P}^{\prime}=\mathrm{p}_{\uparrow}{ }^{\prime}-\mathrm{p}_{\downarrow}{ }^{\prime}$ can be very simply evaluated by considering the partial cross sections describing the probabilities that the neutron is scattered into the same and into the opposite spin state. For the purely magnetic scattering we are interested in, and following standard algebra [11], both of these cross sections, called non-spinflip (nsf) and spin spin-flip (sf), can be given in extremely simple forms [12]:

$$
\begin{aligned}
& \left(\frac{\mathrm{d}^{2} \sigma}{\mathrm{d} \omega \mathrm{d} \Omega}\right)=\left(\frac{\gamma \mathrm{e}}{\mathrm{nc}}\right)^{2} \mathrm{e}^{-2 \mathrm{~W}} \frac{\mathrm{k}^{\prime}}{\mathrm{k}} \int_{-\infty}^{\infty} \frac{\mathrm{dt}}{2 \pi \mathrm{h}} \mathrm{e}^{-i \omega t}\left\langle\mathrm{M}_{\perp}^{2}(-\overrightarrow{\mathrm{q}}, 0) \mathrm{M}_{\perp}^{\mathrm{z}}(\overrightarrow{\mathrm{q}}, \mathrm{t})\right\rangle \\
& \left(\frac{\mathrm{d}^{2} \sigma}{\mathrm{d} \omega \mathrm{d} \Omega}\right)=\left(\frac{\gamma \mathrm{e}}{\hbar c}\right)^{2} \mathrm{e}^{-2 W} \frac{\mathrm{k}^{\prime}}{\mathrm{k}} \int_{-\infty}^{\infty} \frac{\mathrm{d} t}{2 \pi h} e^{-i \omega t}\left\{\mathrm{p}_{\uparrow}\left\langle\mathrm{M}_{\perp}(-\overrightarrow{\mathrm{q}}, 0) \mathrm{M}_{\perp}^{+}(\overrightarrow{\mathrm{q}}, t)\right\rangle+\right. \\
& \left.+p_{\downarrow}<M_{\perp}^{+}(-\vec{q}, 0) M_{\perp}^{-}(\vec{q}, t)>\right\}
\end{aligned}
$$

In these equations the value of the numerical constant re/he is $0.2699 * 10^{-12} \mathrm{~cm}$ if the magnetic moment is measured in $\mu_{B}$ units. The Debye-Waller factor exp (-2W) takes into account in a first approximation that the magnetic atoms participate in the lattice vibrations. It should be dropped if the effect of lattice dynamics on magnetic correlations are treated explicitly, $\mathrm{k}$ and $\mathrm{k}^{\prime}$ are the incoming and outgoing neutron momenta, and $\overrightarrow{\mathrm{M}}_{\perp}$ stands for the component of the total (spin + orbita1) magnetization $\overrightarrow{\mathrm{M}}$ perpendicular to the momentum transfer vector $\overrightarrow{\mathrm{q}}=\overrightarrow{\mathrm{k}}, \overrightarrow{\mathrm{k}}$,

$$
\overrightarrow{\mathrm{M}}_{\perp}=\overrightarrow{\mathrm{M}}-\overrightarrow{\mathrm{q}}(\overrightarrow{\mathrm{q} M}) /|q|^{2}
$$

Furthermore $\quad M^{ \pm}=M^{x} \pm i M^{y}$

and we recall that in this case the direction of the magnetic field on the sample was chosen as the z-axis.

Physically eqs.(3) and (4) have a very simple meaning : correlations in the component of the magnetization $\vec{M}_{\perp}$ parallel to the field direction lead to non-spin-flip scat-. tering, while those perpendicular to the field give rise to spin-flip scattering. (Note that this statement only makes sense if the field and the incoming and outgoing beam polarizations are kept parallel). The well-known complexities of cross section calculations are due to the explicit evaluation of eq. (5) and, first of all, to specific models of spin and orbital magnetization.

The ultimate origin of the magnetic scattering of neutrons is simply the interaction between the neutron magnetic moment and the local magnetic field inside the sample. This is why only the total magnetization matters for neutrons, as it was shown by Tramme 11 [13]. 
Eqs. (3) and (4) can be used for the identification of various magnetic scattering effects, of course. In doing this we have to recall that if the nuclear spins are perfectly randomly oriented, the purely nuclear scattering is fully non-spin-flip, i.e.

$$
P^{\prime}=P
$$

with the exception of the nuclear spin incoherent contribution, for which

$$
P^{\prime}=-\frac{1}{3} P
$$

These two equations are valid not only in the restricted sense of scalar, $\mathrm{h}$ component polarization, but also as vector relations in the general case. It has also to be remembered, that the interplay of several effects, such as magnetism and lattice dynamics, nuclear and magnetic interference scattering, etc. can produce any kind of complexity (e.g. the magnetovibrational scattering can have spin-flip components). Fortunately, these effects are usually weak or can be sorted out with some precaution.

As an example, let us consider magnon scattering in a simple Heisenberg ferromagnet magnetized to saturation in the $z$-direction. It is easy to see that in this case magnon creation and annihilation uniquely correspond to non-vanishing $\left\langle\mathrm{M}^{+} \mathrm{M}^{-}\right\rangle$and $\left\langle\mathrm{M}^{-} \mathrm{M}^{+}\right\rangle$correlations, respectively. If (and only if) we make the magnetic field be parallel to $\vec{q}$, i.e. $\vec{q} / / \mathrm{z}$, then obviously $\left\langle\mathrm{M}^{+} \mathrm{M}^{-}\right\rangle \equiv\left\langle\mathrm{M}_{\perp}^{+} \mathrm{M}_{\perp}^{-}\right\rangle$, and $\left\langle\mathrm{M}^{-} \mathrm{M}^{+}\right\rangle \equiv\left\langle\mathrm{M}_{\perp}^{-} \mathrm{M}_{\perp}^{+}\right\rangle$. Thus in view of eq. (4) the magnon scattering becomes fully spin flip, and if $\mathrm{p}_{\uparrow}=1$ it is exclusively energy gain scattering, while for $\mathrm{p}_{\downarrow}=1$ there is neutron energy loss only. (Note that in eq. (4) $\uparrow$ means neutron magnetic moment parallel to the field, which implies that the neutron spin is opposite to the field. It is easy to remember that in magnetized mirror type polarizers the repulsion of the neutrons by the B-field inside the mirror contributes to the reflection, thus the magnetic polarization of the reflected beam is opposite to the applied field). This means, that for the identification of this type of magnon scattering it is sufficient to use a polarized incoming beam without analyzing the outgoing one, or alternatively, to use an unpolarized incoming beam and to analyse the scattered beam polarization $\mathrm{P}^{\prime}$. These features have been demonstrated several times and to various degrees $[14,15,8]$, but actually rarely used in real experiments in order to single out magnon scattering. The reason for this is that most often other features, like the shape of the dispersion relation, structure factor, magnetic field dependence etc. allow the identification of magnon branches. Problems arise when the magnon and phonon scattering overlap, especially because of finite instrumental resolution. Holden and Stirling used the scattering of polarized neutrons (without polarization analysis of the scattered beam) in the study of $\mathrm{Pd}_{3} \mathrm{Fe}$ alloy [16]. As illustrated in Fig. 1 they were able to identify an unusually broad magnetic contribution over a rather high and structureless background due to 4 phonon branches. The same technique was used by Lowde et al. in the investigation of the high temperature behaviour of $\mathrm{Ni}$ [17]. These authors studied the breakdown of the above simple relation between incoming beam polarization and the sign of the neutron energy change, which shows that at high temperatures the $\mathrm{Ni}$ magnetization is not homogeneously aligned paralle1 to the saturating field.

Another case in which the application of eqs.(3) and (4) is simple is that of magnetically isotropic samples, e.g. paramagnets, polycristalline antiferromagnets, etc. In this case the field applied to the sample should not be too strong in order to avoid the creation of a field induced anisotropy, and in fact it can be just the minimum required as a guide field for maintaining the beam polarization (i.e. typically 1-100 oe, depending on the field geometry). Indeed it is easy to show that if

$$
\left\langle M^{\alpha} M^{\alpha}>=\left\langle M^{\beta} M^{\beta}\right\rangle\right.
$$

and

$$
\left\langle M^{\alpha} M^{\beta}>=0\right.
$$




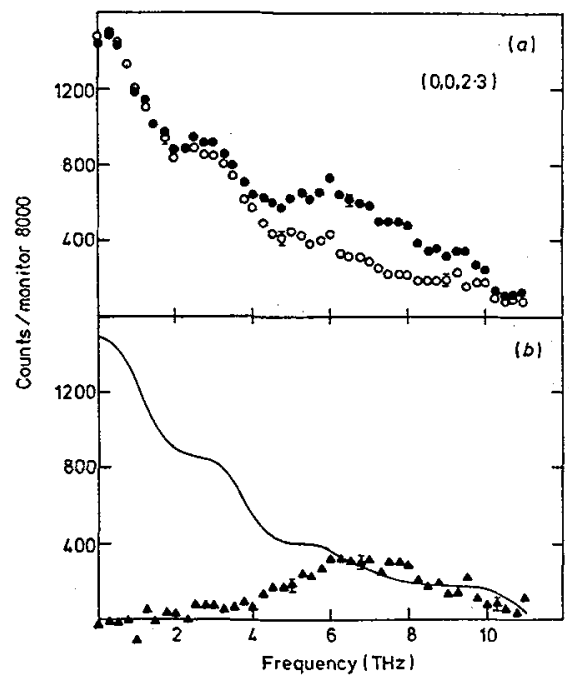

Fig. 1. Identification of magnon scattering in $\mathrm{Pd}_{3} \mathrm{Fe}$. Open and closed circles denote in (a) observed counts in a constant q scan when the neutron polarization is paralle1 $\left(p_{\uparrow}=1\right)$ and antiparallel $\left(p_{\downarrow}=1\right)$ to the magnetic field on the sample. The triangles in (b) show the magnon cross section, and the line the remaining phonon scattering (Holden and Stirling [16]).

(for $\alpha, \beta=\mathrm{x}, \mathrm{y}, z$ ), the total cross section $(d \sigma)_{\mathrm{nsf}}+(d \sigma)_{\text {sf }}$ is independent of the polarization $\left(p_{\uparrow}\right)$ and the $h$ component of the scattered beam polarization for any kind of purely magnetic scattering (magnons, diffuse scattering, etc.) becomes

$$
P^{\prime}=-P \cos ^{2} \alpha
$$

where $\alpha$ is the angle between the momentum transfer $\vec{q}$ and the field direction $\vec{H} / / \vec{p}$. This change of the scattered beam polarization with the field (i.e. incoming beam polarization) direction is in contrast with the behaviour of nuclear scattering effects, eqs.(7) and (8) and it can be used for the unambiguous identification of the magnetic scattering. An example for this is the recent very significant investigation of high temperature paramagnetism at the ILL [18], which is discussed in the paper by Kurt Ziebeck in this volume.

Equation (11) also represents an interesting relation between polarization and inelasticity, as it was pointed out as early as 1965 by the Leningrad group [19]. At a given scattering angle, the direction of the momentum transfer $\vec{q}$ is related to the inelasticity of the scattering, as shown in Fig.2. E.g. if the direction of $\mathrm{P} / / \mathrm{H}$ is chosen to be that of the $\overrightarrow{\mathrm{q}}$ vector of elastic scattering ( $\mathrm{x}$ in Fig.2), $P^{\prime}$ will be equal to $-P$ for elastic scattering, and for inelastic scattering a smaller value of $\left|\mathrm{P}^{\prime}\right|$ will be found which can be rough1y approximated as [19]

$$
P^{\prime}=-P\left(1-\frac{\left\langle\omega^{2}\right\rangle}{\left(2 E_{0} \theta\right)^{2}}\right)
$$

where $\left\langle\omega^{2}\right\rangle$ is the average value of the square of the neutron energy transfer in scattering by angle $\theta$, and $E_{O}$ is the incoming neutron energy. This equation provides a very useful, but rather qualitative indication of the scattering inelasticity, since the quantitative evaluation of $\left\langle\omega^{2}\right\rangle$ requires in general the knowledge of the shape of the scattering function $S(q, \omega)$. The method has been recently used to study the dynamics of critical fluctuations in iron with rather good sensitivity [20] (at very small scattering angles and long neutron wavelengths the denominator in eq. (12) can be as small as 10-20 $\mu \mathrm{eV}$ ). The results of these studies, however, are not very conclusive due to their strong dependence on the model scattering function assumed.

Another fundamental aspect of the problem is that in view of this relation between polarization and inelasticity, the determination of paramagnetic scattering intensities by polarization analysis can only be reliably performed by taking into account the inelasticity of the scattering. With crystal spectrometers, as in Ref. [18], 


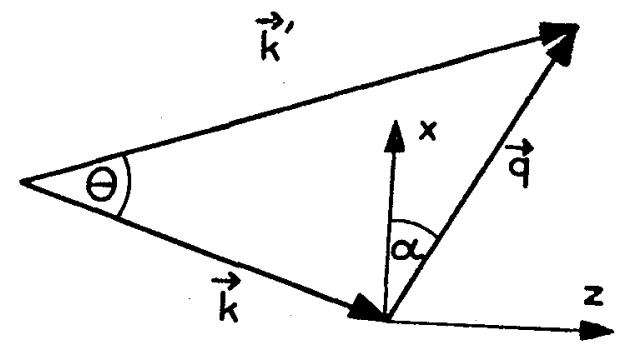

Fig. 2. Definition of the notation used in the text for the description of the polarization behaviour in inelastic scattering at a fixed scattering angle $\theta$.

this is automatically achieved for scattering angles bigger than a few degrees. However, if broad band polarizers or analysers (Iike supermirrors) are used, which provide much better flux for diffuse scattering studies at relatively long neutron wavelengths $(\gtrsim 3 \AA$ ) the inelasticity has to be dealt with explicitly.

Mezei and Murani have introduced [21] a particular scheme to do this, which is based on the determination of $\mathrm{P}^{\prime}$ for three mutually perpendicular direction of $\mathrm{P} / / \mathrm{H}$. For convenience let us consider the coordinate system shown in Fig.2, with the yaxis perpendicular to the scattering plane. Let us denote by $X, Y$ and $Z$ the three differences between the spin-flip and non-spin-flip scattering intensities measured with the incoming beam polarization set subsequently parallel to each of the $x, y$ and $z$ axis, respectively. Thus in view of eq.(11) and remembering that $\bar{q}$ is always perpendicular to the y-axis, we can write

$$
\begin{aligned}
& X=\text { IP }\left\langle\cos ^{2} \alpha\right\rangle+\text { N.S. } \\
& Y=\text { N.S. } \\
& Z=\text { IP }\left\langle\sin ^{2} \alpha\right\rangle+\text { N.S. }
\end{aligned}
$$

where $I$ is the intensity of magnetic scattering, $P$ the overall polarization efficiency of the instrument, the angle $\alpha$ is defined in Fig.2, and N.S. stands for the nuclear scattering contributions. It is easy to see that

$$
I=(X+Z-2 Y) / P
$$

This expression of the total magnetic scattering intensity is not only independent of the distribution of neutron energy change in the scattering at the fixed angle $\theta$, but a1so of the choice of the actual direction of the $x$ and $z$ axis as long as they are in the scattering plane and perpendicular to each other. This is the key to performing this type of polarization analysis using several detectors at different angles at the same time, as it was recently implemented by Otto Schärpf on the D7 instrument at the ILL. Note, that one has to take into account, nevertheless, that the transmission band of supermirror polarizers is still finite (typically limited to neutron energies below $10 \mathrm{meV}$ ) and that if the inelasticity is substantial the fixed scattering angle results do not correspond to constant $q$.

In this scheme of polarization analysis there is a simple way of getting quantitative information about the inelasticity too, if the choice of the $x$ and $z$ direction is that described above in connection with Fig.2 (i.e. $x$ parallel to the direction of the elastic scattering vector). It can be shown that in a good approximation for not too big energy transfers $\left(|\alpha| \leqslant 45^{\circ}\right.$ in Fig.2)

$$
n=\frac{X-Z}{X+Z-2 Y}=\frac{\operatorname{Re} S(q, t)}{S(q)} \quad \text { with } t=\frac{1}{4 E_{0}} \operatorname{ctg}\left(\frac{\theta}{2}\right)
$$


where $s(q, t)$, the so-called intermediate scattering law, is the time dependent Fourier transform of the magnetic scattering function $S(q, \omega)$ and $S(q)$ is the static magnetic structure factor

$$
s(q)=\int_{-\infty}^{\infty} s(q, \omega) d \omega=S(q, t=0)
$$

If for quasielastic scattering the ratio $n$ is close to one $(n \geqslant 0.5)$ our approximation is fairly good, and the scattering at fixed angle $\theta$ corresponds to a roughly constant $|q|$. If $\eta<0.5$, the inelasticity is comparable to $2 \mathrm{E}, \theta$, in which case the scattering at angle $\theta$ spreads over a substantial range of $|q|$. One example for the application of this method is shown in Fig.3. The data represent the time dependence of the spin relaxation in $\mathrm{Cu}-5 \% \mathrm{Mn}$ spin glass alloy (i.e. the right hand side of eq. (15)) at various temperatures. The points at $3 \times 10^{-12}$ sec have been obtained by using eq. (15) and those at longer times are paramagnetic neutron spin echo (see below) results. $6 \AA$ wavelength neutrons were used at $\theta=5^{\circ}$ scattering angle, i.e. $2 \mathrm{E} \theta=0.4 \mathrm{meV}$. In this particular case it was established that

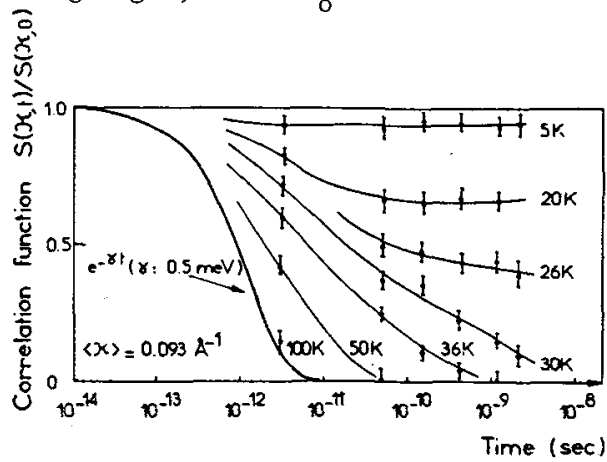

Fig. 3. Spin relaxation in $\mathrm{Cu}(5 \% \mathrm{Mn})$ spin glass at various temperatures as determined by polarization analysis (at $t=$ $3.10^{-12} \mathrm{sec}$ ) and paramagnetic neutron spin echo. The temperature of the cusp in the a.c. susceptibility of the sample is $27.5 \mathrm{~K}$. The lines are guides to the eye. (Mezei and Murani [21])

$\mathrm{S}(\mathrm{q}, t)$ only varies slowly with $\mathrm{q}$, thus there is little difference between constant $|\mathrm{q}|$ and fixed $\theta$ results. Note that the relaxation spreads over several orders of magnitude in time, a peculiar feature found to be characteristic for spin glasses, and the present polarization analysis technique was very instrumental in extending the time domain covered in this experiment.

Another example is the so-called ferromagnetic neutron spin echo (FNSE), which method allows the NSE study of samples in a strong magnetic field [22] (e.g. ferromagnetic samples, or study of field dependent effects). In this case the inelastic spectrum of the difference cross section $(\mathrm{d} \sigma)_{\mathrm{nsf}}-(\mathrm{d} \sigma)_{\mathrm{sf}}$ is directly investigated. An experiment on the inelastic lineshape of diffusive solitons in TMMC in a magnetic field of $4 \mathrm{~T}$ has been recently completed (a preliminary account is given in Ref. [23]).

Finally, there is an old suggestion to use eq.(8) in order to single out unambiguously the nuclear spin incoherent scattering, which purely reflects the self diffusion dynamics. Such experiments were prohibited until recently by the notorious lack of neutron intensity in polarization analysis. This situation has changed now by the application of supermirror polarizers and a conclusive experiment on liquid sodium has been performed last week by Schärpf and Gläser at the ILL.

\section{B) Paramagnetic situations}

The famous Halpern-Johnson equation (1) is a vectorial relation, which does not follow from the above spin-flip and non-spin-flip scattering analysis, i.e. eq.(11). It can be shown [22] that it applies not only to paramagnetic scattering, but also to any kind of magnetic scattering on any kind of macroscopically isotropic system (e.g. magnon scattering or superlattice reflections on possibly anisotropic but ideally polycrystalline antiferromagnets). We define therefore as "paramagnetic" the vector polarization analysis experiment in which the Halpern-Johnson relation applies to the sample. This means that not only the component of $\overrightarrow{\mathrm{P}}$. parallel to $\overrightarrow{\mathrm{P}}$ is considered, but the real direction and magnitude of $\overrightarrow{\vec{F}}$. To do this in practice, the field on the sample must be small so that the precession of the components of 
the polarization perpendicular to the field can be kept track of. (This is why the sample cannot be ferromagnetic).

The basic difference between the Halpern-Johnson equation and the above analysis, eqs.(3) and (4), is that the latter implicitly considers the spin-flip and non-spinflip scattering as independent random processes with no coherence between them. This suggests that the polarization $\vec{P}^{\prime}$ on the average cannot have a component perpendicular to $\overrightarrow{\vec{P}}$. It is very significant that, to the contrary, eq. (1) implies the coherence between these processes, which is the necessary condition for obtaining non-paralle $\overrightarrow{\mathrm{P}}$ and $\overrightarrow{\mathrm{P}}^{\prime}$ via the interference of the $\uparrow$ and $\downarrow$ spin states [24]. This means that it is more correct to think of the scattering process as a single action, whose splitting into e.g. spin-flip and non-spin-flip components is merely a mathematical convenience in certain experimental conditions, but does not correspond to any physical reality.

As a matter of fact the Halpern-Johnson equation (1) has never been explicitly tested as a vector relation, and there is only a single implicit experimental evidence, namely the so-called paramagnetic neutron spin echo (PNSE) [22]. In this technique, routinely used at the ILL for many years (e.g. Refs. [21] and [38]) the change of the neutron polarization in the scattering process as described by eq. (1) is used to produce the echo signal [22]. The interest of the method is that this way nuclear scattering processes do not contribute at all to the measured echo. It has been shown using eq. (1) that the maximum amplitude of the PNSE signal is $50 \%$ of the total magnetic scattering intensity, in agreement with experiment. On the other hand, if the spin-flip and non-spin-flip processes would add incoherently, the scalar eq.(11) should be used instead of eq.(1) and one finds $25 \%$ as the maximum relative amplitude of the PNSE signal. This would imply that on the scale used in Fig. 3 no NSE data point could lie above the ordinate value 0.50 , to the contrary of what was observed.

This represents the only existing test of the Halpern-Johnson equation in its full, vector sense, performed just 40 years after of its publication. At the same time, PNSE is the only example as yet of the category of experiments I define as "paramagnetic".

\section{Antiferromagnetic situations}

This category contains what is left out of the previous two, i.e. vector polarization analysis on macroscopically anisotropic samples which do not obey eq.(1). In these cases $\overrightarrow{\mathrm{P}}^{\prime}$ can be a very complicated function of several vectors [9]. Two experimental examples exist, viz. the diffraction experiment of Alperin [4] and the antiferromagnetic neutron spin echo (AFNSE) method [22], a tested but not yet applied technique for the identification and NSE investigation of magnetic scattering in anisotropic samples.

\section{Neutron polarization as a tool for measuring inelasticity}

In the investigation of inelastic scattering both the preparation of the beam impinging on the sample and the analysis of the scattered beam requires some sort of action on the neutrons (such as chopping or monochromatization). The action which represents the minimum intervention with the neutron state is the modification of the direction vector of the neutron spin. For example, the energy of interaction involved is of the order of the Zeeman splitting, which can be as little as $10^{-10} \mathrm{eV}$ (corresponding to $\sim 8$ oe field). Since neutron polarizers can distinguish between different spin states with a very high efficiency ( $>95 \%$ is common), the minimal action on the neutron spin can be transformed into a drastic effect on the beam, e.g. changing its intensity by a factor of 40 or more. In what follows I will review the various techniques of neutron spin modulation, which have been used or proposed for the investigation of scattering inelasticity.

\section{A. The Drabkin monochromator}

Even the most elementary operation in neutron scattering, i.e. the production of a 
monochromatic beam can be achieved by the mere action of magnetic fields on the neutron spin, without any mechanically moving parts, as suggested by Drabkin in 1962 [25] and shown in Fig.4. The initially polarized beam traverses a homogeneous field region $\mathrm{H}_{\mathrm{O}}$ along an $\mathrm{Al}$ foil folded in a wavy pattern and carrying a d.c. current. The small field $\mathrm{H}_{1}$ produced by the foil oscillates in space along the neutron trajectory with the periodicity of the fold $d$. For the neutron with velocity $v$ the $\mathrm{H}_{1}$ field appears to oscillate in time, and if this oscillation frequency is close to the Larmor frequency, i.e.

$$
\frac{\mathrm{v}}{\mathrm{d}}=\gamma_{\mathrm{L}_{\mathrm{O}}} \mathrm{H}_{\mathrm{O}}
$$

(where $\gamma_{\mathrm{L}}=2.916 \mathrm{kHz} / \mathrm{Oe}_{\mathrm{e}}$ ), the spin of the neutron will be reversed, assuming that $\mathrm{H}$ has the right magnitude. The spin flipped, monochromatic neutrons can be separated from the rest of the beam by a second polarizer. Equation (17) shows that the selected neutron velocity $\mathrm{v}$ can simply be tuned by changing the field $\mathrm{H}_{\mathrm{O}}$.

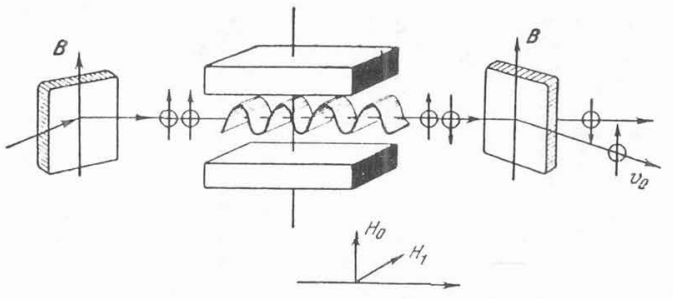

Fig. 4. Magnetic monochromator for polarized neutrons (Drabkin [25]).

The relative monochromaticity of the beam, $\Delta v / v$ is the inverse of the number of foil periods traversed. Such a monochromator system is actually in operation in Leningrad [26], it is used in a small angle scattering instrument. In order to improve the discrimination ratio between selected and discarded neutrons and to eliminate the interference pattern like fringes of the monochromatized velocity spectrum, two such devices of somewhat different band widths are used one after the other. One of the interests of this method is that if a very large number of periods could be used $\left(\sim 10^{3}\right)$ the produced beam monochromaticity could be very high and variable by simply activating only a part of the folded foil.

\section{B. Spin-flip-chopper}

The electronically gated beam chopping device is an old dream in neutron time-offlight spectroscopy, partly because of the speed of mechanical choppers is limited, (minimum pulse length $\sim 20 \mathrm{\mu sec}$ ) part1y because of the flexibility of pulse pattern offered by electronic chopping. There is no fully satisfactory solution known as yet. One of the possible approaches, pioneered by the Vienna group [27] is illustrated in Fig. 5. The activation of the spin flipper placed between the two polarizers allows to shut the beam off. Such a device has a very satisfactory fast

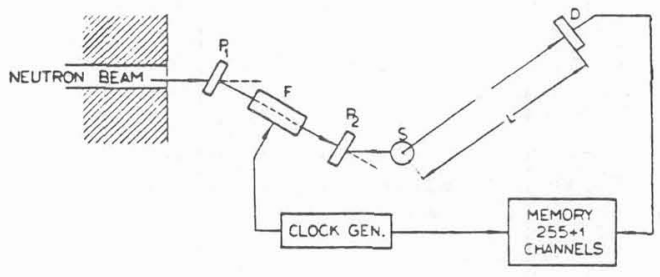

Fig. 5. Scheme of a time-of-flight spectrometer using spin-flip-chopper (after Pàl et. al. [29]):

switching time, in particular by using the d.c. flipper coil introduced by the author [7] as short as $5 \mu \mathrm{sec}$ duration neutron bursts have been achieved by Badurek [28]. A serious shortcoming of the spin-flip-chopper is that the beam-on - beam-off intensity ratio is insufficient (typically $50: 1$ ) for general use in time-of-flight 
spectroscopy. This is a lesser drawback if not single neutron bursts, but a statistical pulse pattern is used, as first demonstrated by the Budapest group in 1968 [29]. In correlation spectroscopy the much more ideally square lineshape in a larger beam cross section and the flexibility of control is a very real advantage of spin flip choppers. Due to the lack of high intensity polarizers this technique was not made practical use of as yet. The situation might change soon with the application of powerful supermirror polarizer systems [30], as it was recently tested on the D7 spectrometer by 0tto Schärpf.

\section{Spin-flip-modulation}

In the investigation of time-of-flight spectra of magnetic scattering effects with polarization dependent cross section (eg. magnons in ferromagnets, as discussed above) there is no need to chop the incoming beam. It is sufficient to modulate the incoming polarization in time instead. Following a sudden inversion of the neutron spin direction the corresponding change in the scattered beam intensity will be observable at the detector with the time delay corresponding to the neutron time-of-flight. This method was first demonstrated around 1968 at Kjeller by Steinsvoll and Virjo [15] using $35 \mu \mathrm{sec}$ spin flip bursts in order to observe the magnon response in an iron single crystal, see Fig. 6.

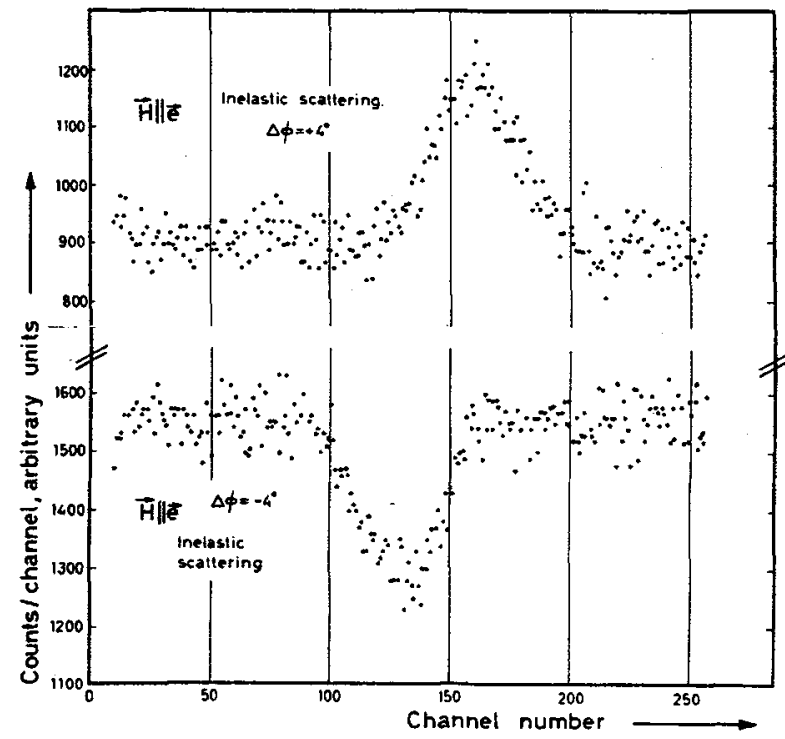

Fig. 6. Observation of magnon creation (upper part) and annihilation in an $\mathrm{Fe}$ single crystal by the measurement of the timeof-flight response of the counting rate following $35 \mu \mathrm{sec}$ duration spin-flip bursts. Note the opposite sign of the modulation in the two processes, cf. eq. (4). (after Steinsvoll and Virjo [15])

(The experimental arrangement was similar to that shown in Fig.7 below). This technique also suffered from the low polarized fluxes available and therefore sköld suggested [31] that the flipper should be activated in a statistical sequence rather than in single bursts. Mezei and Pellionisz were the first to realize statistical spin-flip-modulation spectroscopy experimentally in 1971 [32] and in fact this is the only technique of those we have just discussed in points $B$ and $C$ which has really been useful in practice. The reason for this is that the experimental configuration is just that of an ordinary polarized neutron diffractometer (cf. Fig.7), and switching the flipper on and off in a rapid statistical sequence is simply a special way of sharing the total measuring time between the flipper-off and flipper-on counts. Thus without losing either neutron intensity or information, the statistical spin-flip modulation allows to obtain in addition the time-offlight spectrum of the spin dependent part of the cross section, as illustrated in the right hand side of Fig.7. The very rough time-of-flight analysis in these old data on diffuse scattering on a dilute $\mathrm{Fe}(\mathrm{Mn})$ alloy is perfectly sufficient to separate three contributions : the one due to the $\lambda / 2$ contamination of the incoming beam, the inelastic magnetovibrational scattering and the elastic diffuse scattering. 

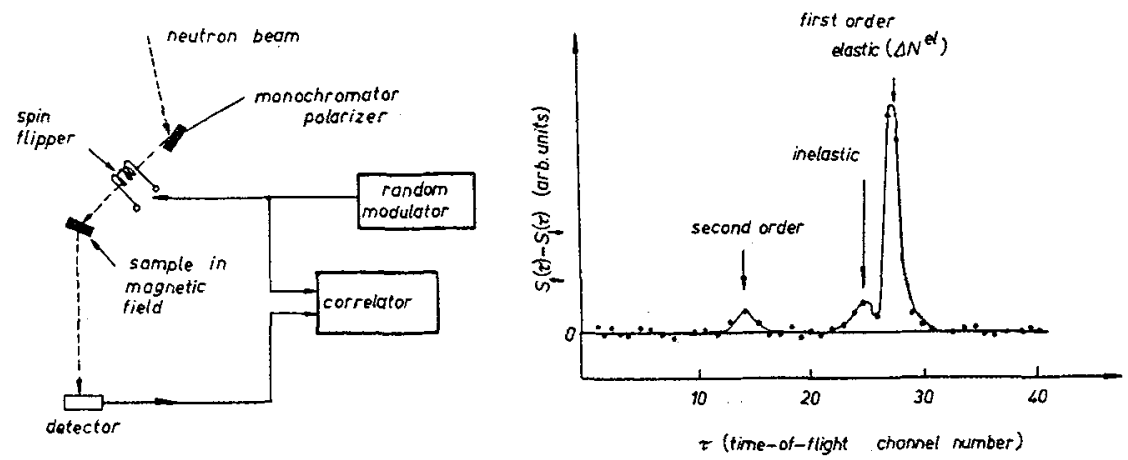

Fig. 7. Experimental set-up for statistical spin-flip-modulation spectroscopy and a sample result showing the time-of-flight spectrum of the polarization dependent scattering intensity of a dilute Fe(Mn) alloy (Mezei and Pellionisz [32]).

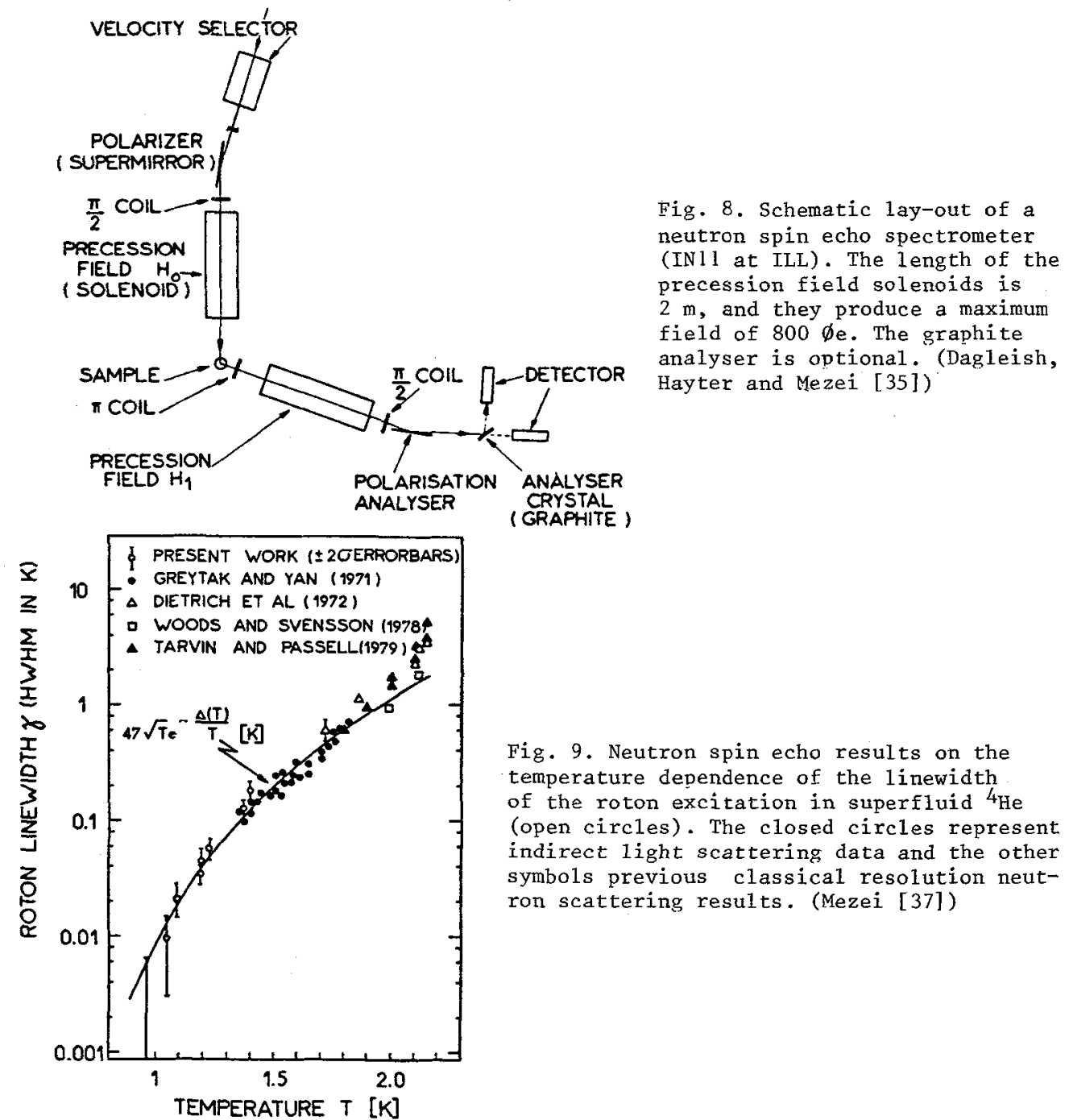


It is obvious that such a separation of the parasitic contributions is indispensable e.g. in the study of the elastic diffuse scattering especially at room temperature or above [33]. Unfortunately, the literature abounds in results which are spurious or uncertain in this respect. The method has also been applied for the investigation of magnon excitations in amorphous ferromagnets by Mook [34].

\section{Neutron Spin Echo}

This technique represents a rather special and unique approach to inelastic neutron scattering based upon the determination of the velocity change of individual neutrons [7]. This is in contrast to the other inelastic scattering techniques concerned with the determination of the change of the average velocities of the whole incoming and outgoing beams. In NSE the initially polarized incoming beam is made to perform Larmor precessions while flying through a well defined magnetic field region $H_{O}$, which allows each individual neutron to clock its own velocity (cf. Fig.8). After scattering the neutrons traverse another magnetic field regions precessing effectively in the opposite sense, which serves for the comparison of the outgoing and incoming velocities for each neutron independently. Thus uniquely high resolutions can be achieved with poorly monochromatic and therefore intense beams. For example the IN11 spectrometer [35] at the ILL can reveal 2 neV inelastic 1 inewidths with an incoming beam of $1.3 \mathrm{meV}$ energy and $20 \%$ monochromaticity.

As a matter of fact, the NSE work represents the majority of inelastic scattering experiments performed by now using polarized neutrons. In more than half of these cases, the sample was not magnetic at a11, in others the inherent use of polarized neutrons in NSE served both for the inelastic analysis and the identification of the magnetic scattering effects. This field has been recently reviewed in the literature [36], and I will only mention two representative results here which illustrate the progress of neutron scattering in the last decade.

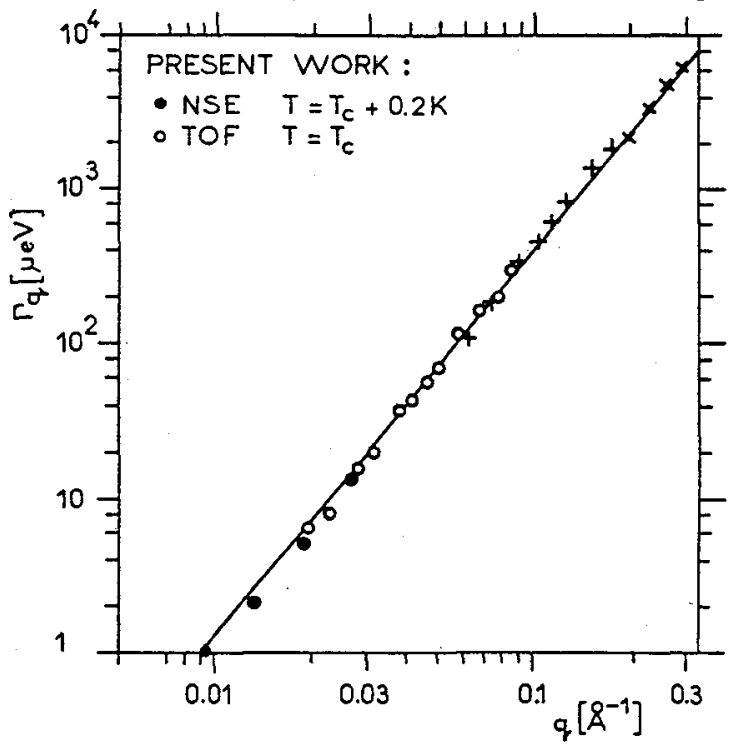

Fig. 10. Wavenumber dependence of the quasielastic linewidth of the critical fluctuations in $\mathrm{Fe}$ at the Curie point $\mathrm{T}=1044 \mathrm{~K}$. The circles represent recent neutron spin echo and time-of-flight results, and the crosses show the previous neutron triple-axis spectrometer data. The straight line indicates the $\mathrm{q}^{5 / 2}$ law (Mezei [38]).

The first example, shown in Fig.9 concerns the line width of the roton excitation (energy $\Delta=742 \mu \mathrm{eV}=8.62 \mathrm{~K}$ ) in superfluid ${ }^{4} \mathrm{He}$, a non-magnetic sample [37]. The previous neutron scattering and light scattering data were in reasonable agreement with the theoretical prediction of Landau and Khalatnikov (continuous line) but further light scattering results (not shown) indicated a constant linewidth below $1.2 \mathrm{~K}$. By the some 50 fold improved resolution of NSE compared with the previous neutron experiments the theoretical prediction could be verified below $1.2 \mathrm{~K}$ too showing that the indirect light scattering results were influenced by other effects.

The other example is a magnetic problem, the dynamics of critical fluctuations in Fe 
at the Curie point [38]. Fig. 10 shows the measured quasielastic linewidths as a function of the momentum transfer at $\mathrm{T}=\mathrm{T}_{\mathrm{c}}$. The early triple axis results could be extended by two orders of magnitude in energy resolution by using advanced time-offlight and paramagnetic NSE methods (IN5 and IN11 spectrometers, respectively, at ILL). All these data join nicely together into the predicted power 1 aw $\Gamma \alpha q^{5 / 2}$, covering four orders of magnitude in energy. In view of recent speculations based on indirect (and misinterpreted) hyperfine field evidence, this is a totally unexpected result. This example underlines the pivotal role the uniquely direct neutron scattering, and in particular polarized neutron results play in magnetism research.

\section{E. Neutron Spectral Modulation (NSM)}

This latest development in high resolution spectroscopy, proposed by Ito [39] combines features of NSE and statistical spin-flip modulation. Larmor precessions between two polarisers are used to modulate the incoming, relatively broad neutron velocity distribution by a sine wave, producing a comblike spectrum with several sharp peaks. The scattered beam velocity, on the other hand, is determined by the neutron time-of-flight, making use of the statistical spin-flip modulation of the incoming beam pattern. The method promises high energy resolution, intermediate between NSE and classical crystal spectrometers with the advantage of providing full time-of-flight spectra in a large solid angle.

\section{Conclusion}

Polarized neutrons have been applied in inelastic scattering studies with great benefits. In one kind of applications the neutron polarization is used to identify unambiguously the magnetic scattering effects. The examples are not numerous, but recent developments in neutron polarizers allow the extension of such methods to the study of diffuse scattering effects. In this case, as recent results illustrate, such an identification is often indispensable, and a multiplication of experiments with polarized neutrons is to be expected. The other type of utilization of neutron polarization is the use of spin modulation for the investigation of the inelasticity itself for any kind of scattering. Examples, first of all in NSE spectroscopy, abound, and further developments are in progress. Polarized neutrons proved to be unique tools in opening up new possibilities in the more precise analysis of scattering inelasticity.

\section{References}

1 Halpern O., Johnson M.H., Phys. Rev. 55 (1939) 898

2 Rekveldt M. Th., J. de Physique 32 (1971) C579; Rekveldt M. Th., Z. Phys. 259 (1973) 391

3 Okorokov A.I., Runov V.V., Volkov V.I., Gukasov A.G., ZhETF 69 (1975) 590, (Soviet Phys. JETP 42 (1976) 300)

4 Alperin H., Proceedings ICM-73 (Moscow, 1973) Vo1. III, p. 128

5 Mezei F., Magn. Magn. Mat. 1973, Boston,AIP Conf. Proc. N 18 (New York, 1974) p. 406

6 Tasset F., Private Communication

7 Mezei F., Z. Phys. 255 (1972) 146

8 Moon R.M., Riste T., Koehler W.C., Phys. Rev. 181 (1969) 920

9 See Izyumov Yu A., Maleev S.V., Zh. Eksp. i. Teor. Fiz. 4 I (1961) 1644

(Sov. Phys. - JETP 14 (1962) 1168); Blume M., Phys. Rev. 130 (1963) 1670;

Schermer R.I., Blume M., Phys. Rev. 166 (1968) 554

10 Halpern O., Holstein T., Phys. Rev. 59 (1941) 960

11 See Marsha11 W., Lovesey S.W., Theory of Neutron Scattering. (Oxford, 1971)

12 Mezei F., Radhakrishna P., Ann. Phys. 7 (1972) 269

13 Trammel1 G.T., Phys. Rev. 92 (1953) 1387 
14 Samuelsen E.J., Riste T., Steinsvoll 0., Phys. Lett. 6 (1963) 47

15 Steinsvol1 0., Virjo A., Neutron Inelastic Scattering 1968 (IAEA, Vienna 1968) Vol. II, p.395

16 Holden T.M., Stirling W.G., J. Phys. F : Metal Phys., 9 (1977) 1901

17 Lowde R.D., Moon R.M., Pagonis B., Perry C.H., Sokoloff J.B., Vaughan-Watkins R.S., Wiltshire M.C.K., Crangle J., submitted to J. Phys. C

18 Brown P.J., Deportes J., Givord D., Ziebeck K.R.A., J. App1. Phys. 53 (1982) 1973

19 Drabkin G.M., Zabidarov E.I., Kasman Ya. A., Okorokov A.I., ZhETF Pis. Red. 2 (1965) 541 (JETP Lett. 2 (1965) 336); Maleev S.V., ZhETF Pis. Red. 2 (1965) 545 (JETP Lett. 2 (1965) 338)

20 Hetzelt M., Heidemann A., Nucl. Inst. Meth. 133 (1976) 51; Okorokov A.I., Runov V.V., Gukasov A., Nucl. Inst. Meth., $1 \overline{57}$ (1978) 487 and these proceedings

21 Mezei F., Murani A.P., J. Magn. Magn. Mat., 14 (1979) 211

22 Mezei F., Neutron Spin Echo, Mezei F., Editeur (Springer Verlag, Heidelberg 1980) p.3

23 Boucher J.P., et al., Proc. Yamada Conference VI, Sept. 1982, Hakone Japan (in press)

24 Mezei F., Imaging Processes and Coherence in Physics, Schlenker M. et al. Editors (Springer Verlag, Heidelberg 1980) p.282

25 Drabkin G.M., ZhETF 43 (1962) 1107 (Sov. Phys. JETP, 16 (1963) 781L)

26 Agamal'an M.M., Drabkin G.M., Otchik Ya. M., Leningrad Nuclear Phys. Inst. preprint $\mathrm{N}^{\circ} 470$ (1979)

27. Rauch H., Harms J., Moldaschl H., Neutron Ineiastic Scattering 1968 (IAEA, 1968) Vo1. II, p.387

28 Badurek G., Nucl. Inst. Meth. 189 (1981) 543

29 Pal L., Kroo N., Pellionisz P., Szlavik F., Vizi I., Neutron Inelastic Scattering 1968 (IAEA, Vienna 1968) Vol. II, p.407

30 Scharpf 0., Neutron Scattering Symp., Argonne 1981, AIP Conf. Proc. $\mathrm{N}^{\circ} 89$ (New York, 1982) p. 182

31 Skold K., Nucl. Inst. Meth., 63 (1968) 347

32 Mezei F., Pellionisz P., Nucl. Inst. Meth., 99 (1972) 613

33 Mezei F., Neutron Scat. Conf. Proc., Moon R., Editeur (Oak Ridge, 1976) Vol. II, p.670

34 Mook H.A., Tsuei C.C., Phys. Rev. B16 (1977) 2184

35 Dagleish P.A., Hayter J.B., Mezei F., Neutron Spin Echo, Mezei F., Editor, (Springer Verlag, Heidelberg 1980) p.66

36 Mezei F., Neutron Spin Echo, Mezei F., Editor (Springer Verlag, 1980); Mezei F., Neutron Scatt. Symp. Argonne 1981, AIP Conf. Proc. N 89 (New York 1982) p. 379

37 Mezei F., Phys. Rev. Lett., 44 (1980) 1602

38 Mezei F., Phys. Rev. Lett., 49 (1982) 1096

39 Ito Y., Nishi M., Motoya K., Nucl. Inst. Meth. 198 (1982) 497. 